\title{
The process of Coulomb dissociation of weakly bound relativistic hypernuclei within the two-cluster model
}

\author{
Valery V. LYUBOSHITZ* \\ Joint Institute for Nuclear Research (Dubna, Russia) \\ E-mail: Valery.Lyuboshitz@jinr.ru \\ Vladimir L. Lyuboshitz \\ Joint Institute for Nuclear Research (Dubna, Russia)
}

\begin{abstract}
Using the analogy with the problem of ionization and excitation of atoms at the propagation of relativistic charged particles through matter, the process of Coulomb dissociation of weakly bound relativistic nuclei and hypernuclei is theoretically investigated in the framework of the two-cluster deuteron-like model. On the basis of the general formula for the total cross-section of excitation and dissociation of a relativistic nucleus in the Coulomb field, explicit analytical expressions for the total cross-section of Coulomb disintegration of weakly bound systems are derived, taking into account also the additional correction terms connected with the finite size of the target nucleus. Numerical estimates for the Coulomb dissociation of relativistic hypernuclei ${ }^{3} H_{\Lambda}$ and ${ }^{6} \mathrm{He}_{\Lambda}$ are performed, and it is demonstrated that for these cases ( especially - for ${ }^{6} \mathrm{H} e_{\Lambda}$ ) the corrections due to the finite size of the target prove to be rather essential.

It is shown that in the limit of very small binding energies $\varepsilon$ bin the cross-section of Coulomb dissociation increases inversely proportionally to $\varepsilon_{\text {bin }}$, and - due to such a sharp dependence upon the binding energy - the experimental measurement of this cross-section in the case of weakly bound relativistic nuclei and hypernuclei allows one to determine the values of binding energy for these systems.
\end{abstract}

XVII International Conference on Hadron Spectroscopy and Structure 25-29 September, 2017

University of Salamanca, Salamanca, Spain

${ }^{*}$ Speaker. 


\section{Excitation and disintegration of relativistic nuclei in the Coulomb field of a point-like charge}

As it was shown previously in the paper [1], where the process of excitation and disintegration of relativistic nuclei and hypernuclei was investigated using the direct analogy with the problem of ionization and excitation of atoms at the propagation of relativistic charged particles through matter [2], the total cross-section of excitation and dissociation of a relativistic nucleus in the field of an immovable Coulomb centre with the charge $Z e$ can be finally presented in the following form ( see also the more detailed consideration, e.g., in $[3,4]$ ) :

$$
\sigma=\frac{4 \pi(Z \alpha)^{2}}{3 v^{2}}\left\langle 0\left|\left(\sum_{p} \mathbf{r}_{p}\right)^{2}\right| 0\right\rangle\left[\ln \left(\frac{\gamma^{2} v^{2}}{\varepsilon_{\mathrm{bin}}^{2}\left\langle 0\left|\left(\sum_{p} \mathbf{r}_{p}\right)^{2}\right| 0\right\rangle}\right)-2 A+B-v^{2}\right]
$$

Here $\hbar=c=1, \alpha=e^{2}=1 / 137$ is the electromagnetic constant, $v=|\mathbf{v}|$ is the velocity of the projectile nucleus in the rest frame of the Coulomb centre (i.e. in the laboratory frame ), $\gamma$ is the Lorentz factor, $\varepsilon_{\text {bin }}$ is the binding energy of the projectile nucleus and $|0\rangle$ is its ground state .

In Eq. (1.1), the summation is performed over the coordinates of all the protons in the projectile nucleus. The constant $A$ involves the dependence of the minimal momentum transfer, at the transition to excited states of the projectile nucleus $|n\rangle$, upon the excitation energy $\varepsilon_{n 0}>\varepsilon_{\text {bin }}$ ( for more details, see $[3,4]$ ). Meantime, the constant $B$ describes the contribution of comparatively large transfers of transverse momentum. Calculations lead to the following expressions:

$$
\begin{gathered}
B=-3 \int_{0}^{\infty} \ln y \frac{d}{d y}\left(\frac{G(y)}{y}\right) d y, \quad y=\mathbf{q}_{\perp}^{2}\left\langle 0\left|\left(\sum_{p} \mathbf{r}_{p}\right)^{2}\right| 0\right\rangle, \\
G(y)=\sum_{n \neq 0}\left|\left\langle n\left|\sum_{p} \exp \left(-i \mathbf{q}_{\perp} \mathbf{r}_{p}\right)\right| 0\right\rangle\right|^{2}=\left\langle\left. 0|| \sum_{p} \exp \left(-i \mathbf{q}_{\perp} \mathbf{r}_{p}\right)\right|^{2} \mid 0\right\rangle-\left|\left\langle 0\left|\sum_{p} \exp \left(-i \mathbf{q}_{\perp} \mathbf{r}_{p}\right)\right| 0\right\rangle\right|^{2},
\end{gathered}
$$

where $\mathbf{q}_{\perp}$ is the transverse momentum transferred to the nucleus .

For the function $G(y)(1.3)$, at $y \ll 1$ we have: $G(y) \approx y / 3$. Meantime, at $y \gg 1$ we obtain: $G(y) \rightarrow z$, where $z$ is the number of protons in the projectile nucleus.

\section{Contribution of finite sizes of the target nucleus}

Taking into account that the target nucleus is not point-like, one should subtract the correction term $\Delta B$ from the constant $B$ in Eq. (1.1). This correction term has the following structure :

$$
\Delta B=3 \int_{0}^{\infty} \frac{G(y)(1-H(y))}{y^{2}} d y ; \quad H(y)=\frac{1}{Z^{2}}\left|\left\langle 0^{\prime}\left|\sum_{p} \exp \left(-i \mathbf{q}_{\perp} \mathbf{r}_{p}^{\prime}\right)\right| 0^{\prime}\right\rangle\right|^{2}
$$

Here $G(y)$ is determined by Eq. (1.3) as before, and $H(y)$ is the square of the electromagnetic formfactor of the ground state of the target nucleus $\left|0^{\prime}\right\rangle$; in doing so, $\mathbf{r}_{p}^{\prime}$ is the coordinate of a 
proton in the target nucleus. For the uniform distribution of protons over the volume of the target nucleus, we have:

$$
H(y)=9\left(\frac{\sin x}{x^{3}}-\frac{\cos x}{x^{2}}\right)^{2}, \quad x=\sqrt{y} \frac{R_{\mathrm{tag}}}{\sqrt{\left\langle 0\left|\left(\sum_{p} \mathbf{r}_{p}\right)^{2}\right| 0\right\rangle}},
$$

$R_{\mathrm{tag}}$ is the radius of the target nucleus. It is obvious that $H(0)=1$.

It is clear from Eq. (1.1) that in the case of relativistic nuclei with small binding energies the principal contribution into the cross-section of the Coulomb dissociation is provided by the logarithmic term being proportional to $\ln \left[\gamma^{2} v^{2} / \varepsilon_{\text {bin }}^{2}\left\langle 0\left|\left(\sum_{p} \mathbf{r}_{p}\right)^{2}\right| 0\right\rangle\right]$.

\section{Weakly bound systems. Two-cluster model}

Now we will consider the Coulomb disintegration of the "friable", deuteron-like nuclei consisting of two clusters ( charged and neutral ), the average distance between which is significantly larger than the radius of the force action and the sizes of the clusters themselves ( see also, e.g., our works [3,4] ). In this case the excited bound states are absent, and the normalized wave function of the ground state, corresponding to the zero orbital momentum, has the following form:

$$
\phi_{0}(r)=\frac{1}{\sqrt{2 \pi \rho}} \frac{\exp (-r / \rho)}{r}, \quad \rho=\left(2 \frac{m_{1} m_{2}}{M} \varepsilon_{\text {bin }}\right)^{-1 / 2},
$$

where $r=|\mathbf{r}|$ is the distance between the clusters .

Here $m_{1}$ and $m_{2}$ are the masses of the charged and neutral clusters, respectively, $M=$ $m_{1}+m_{2}$ is the mass of the deuteron-like nucleus, $\varepsilon_{0}=\left(-\varepsilon_{\text {bin }}\right)$ is the energy of its bound state. In the given case the quantity $\left\langle 0\left|\left(\sum_{p} \mathbf{r}_{p}\right)^{2}\right| 0\right\rangle$ can be explicitly determined:

$$
\left\langle 0\left|\left(\sum_{p} \mathbf{r}_{p}\right)^{2}\right| 0\right\rangle \approx 4 \pi z^{2}\left(\frac{m_{2}}{M}\right)^{2} \int_{0}^{\infty} \phi_{0}^{2}(r) r^{4} d r=z^{2}\left(\frac{m_{2}}{M}\right)^{2} \frac{\rho^{2}}{2}=\frac{1}{4} z^{2} \frac{m_{2}}{m_{1} M \varepsilon_{\text {bin }}},
$$

where $z$ is the number of protons in the charged cluster.

Using Eqs. (1.3) and (3.1), we obtain the analytical expression for the function $G(y)$ :

$$
G(y)=z^{2}\left(1-\left|\int \phi_{0}^{2}(r) \exp \left(-i \mathbf{q}_{\perp} \mathbf{r} \frac{m_{2}}{M}\right) d^{3} \mathbf{r}\right|^{2}\right)=z^{2}\left(1-\frac{2 z^{2}}{y}\left[\arctan \left(\sqrt{\frac{y}{2 z^{2}}}\right)\right]^{2}\right) .
$$

Here, in accordance with Eq. (3.2),

$$
y=\frac{1}{4} \mathbf{q}_{\perp}^{2} z^{2} \frac{m_{2}}{m_{1} M \varepsilon_{\text {bin }}} .
$$

At very small binding energies, the effective radius $\rho$ of the projectile nucleus (see Eq. (3.1)) considerably exceeds the target radius $R$ ( $\rho \gg R$ ). In doing so, in the first approximation we may take $H(y)=1$, considering the target nucleus as a point-like Coulomb centre. Substituting Eq. (3.3) into Eq. (1.2), we obtain the following value for the constant $B$ :

$$
B=-3 z^{2} \int_{0}^{\infty} \ln y \frac{d}{d y}\left(\frac{1}{y}-\frac{2 z^{2}}{y^{2}}\left[\arctan \left(\sqrt{\frac{y}{2 z^{2}}}\right)\right]^{2}\right) d y=\ln 2 z^{2}+C,
$$


where $C \approx 0.316$ ( see [1]).

Meantime, taking into account that at small binding energies all excited states belong to the continuous spectrum, for a two-cluster system with the wave function $\phi_{0}(r)$ (see Eq. (3.1)) the constant $A$ in Eq. (1.1) is determined by the integral:

$$
A=\frac{16}{\pi} \int_{0}^{\infty} \frac{t^{3 / 2}}{(1+t)^{4}} \ln (1+t) d t \approx 1.218 .
$$

Finally, Eq. (1.1) leads to the following result for the total cross-section of Coulomb disintegration of the weakly bound two-cluster nuclei, taking into account also the correction term $\triangle B(Z)$ connected with the finite size of the target nucleus:

$$
\sigma=\frac{\pi}{3}(Z \alpha)^{2} z^{2} \frac{m_{2}}{v^{2} M m_{1} \varepsilon_{\text {bin }}}\left[\ln \left(\frac{8 \gamma^{2} v^{2} M m_{1}}{m_{2} \varepsilon_{\text {bin }}}\right)-(2 A-C)-v^{2}-\Delta B(Z)\right] .
$$

Here $2 A-C \approx 2.12$; the quantity $\Delta B(Z)$ in Eq. (3.7) is determined according to Eq. (2.1).

It is well seen from Eq. (3.7) that in the limit of very small binding energies $\varepsilon_{\text {bin }}$ the crosssection of Coulomb disintegration increases inversely proportionally to $\varepsilon_{\text {bin }}$; in so doing, the $\log$ arithmic term inside the square brackets essentially exceeds the other ones. Measuring experimentally the Coulomb dissociation cross-section $\sigma$ for weakly bound nuclei and hypernuclei, one can determine, in principle, the value of the binding energy $\varepsilon_{\text {bin }}$ for these nuclei.

\section{Projectile nuclei with small binding energies: corrections due to the finite radius of the target nucleus}

Due to the correction term $\Delta B(Z)$, the cross-section of Coulomb dissociation $\sigma$ decreases as compared with the case of the point-like target $(\Delta \sigma<0)$. Substituting Eqs. (3.3), (2.2) into Eq. (2.1), we obtain the quantity $\Delta B(Z)$ in the case of two-cluster projectile nuclei as the integral

$$
\Delta B(Z)=\int_{0}^{\infty} \frac{s^{2}-(\arctan s)^{2}}{s^{5}}\left[1-9\left(\frac{\sin x}{x^{3}}-\frac{\cos x}{x^{2}}\right)^{2}\right] d s,
$$

where, in accordance with the formula for $x$ ( see Eq. (2.2) ) and Eq. (3.4),

$$
s=\frac{1}{z} \sqrt{\frac{y}{2}}, \quad x=\chi s, \quad \chi=2 \frac{R_{\mathrm{tag}}}{\rho} \frac{M}{m_{2}}=2 \sqrt{2} R_{\mathrm{tag}} \sqrt{\frac{M m_{1}}{m_{2}} \varepsilon_{\mathrm{bin}}} .
$$

The dependence of the correction term $\Delta B$ on the parameter $\chi$ is studied in detail, e.g., in $[3,4]$. The corresponding correction to the Coulomb dissociation cross-section is

$$
\Delta \sigma=-\frac{\pi}{3}(Z \alpha)^{2} z^{2} \frac{m_{2}}{v^{2} M m_{1} \varepsilon_{\text {bin }}} \Delta B(Z) .
$$

It should be emphasized that, owing to the dependence of the quantity $\Delta B$ upon the radius of the target nucleus ( for heavy nuclei $R_{\mathrm{tag}} \approx 1.5 Z^{1 / 3}$ ) the total cross-section of Coulomb dissociation $\sigma$ gets a certain deviation from the pure dependence $\sim Z^{2}$.

Let us note that, for a given projectile nucleus, at charges of target nuclei in the interval $Z=$ $50 \div 100$ the dependence of $\Delta B$ on $\chi$ is almost linear $\left(\Delta B \approx b_{\mathrm{pr}} \chi\right)$. As a result, the quantities $\Delta B, \Delta \sigma$ are proportional, respectively, to the factors $Z^{1 / 3}, Z^{7 / 3}: \Delta B \sim Z^{1 / 3}, \quad \Delta \sigma \sim Z^{7 / 3}$. In so doing, the "effective" charge, determining the cross-section of the Coulomb dissociation, is $Z_{\text {eff }}=Z\left(1-a_{\mathrm{pr}} Z^{1 / 3}\right)^{1 / 2}$. The coefficients $b_{\mathrm{pr}}$ and $a_{\mathrm{pr}}$ depend on the concrete projectile nucleus. 


\section{Calculations of the cross-section of Coulomb dissociation for the hypernuclei ${ }^{3} H_{\Lambda}$} and ${ }^{6} H e_{\Lambda}$

1. Let us consider the process of Coulomb dissociation of the hypernucleus ${ }^{3} H_{\Lambda}$ into the deuteron and the $\Lambda$-particle: ${ }^{3} H_{\Lambda}+Z \rightarrow d+\Lambda+Z\left(z=1, M=M\left({ }^{3} H_{\Lambda}\right)=2993.6 \mathrm{MeV} / c^{2}\right.$; $\left.m_{1}=m_{d}=1878 \mathrm{MeV} / c^{2} ; m_{2}=m_{\Lambda}=1115.7 \mathrm{MeV} / c^{2}\right)$.

According to the experimental data, the binding energy of the $\Lambda$ hyperon in the hypernucleus ${ }^{3} H_{\Lambda}$ is $\varepsilon_{\text {bin }}^{(\Lambda)}=(0.01 \pm 0.07) \mathrm{MeV}$ [5], or $\varepsilon_{\text {bin }}^{(\Lambda)}=(0.15 \pm 0.07) \mathrm{MeV}$ [6]. Taking $\gamma=6$ and $\varepsilon_{\text {bin }}^{(\Lambda)} \approx 0.08 \mathrm{MeV}$, we obtain:

$$
\chi \approx 0.43 Z^{1 / 3} ; \quad \Delta B \approx 0.63 \chi ; \quad Z_{\text {eff }}^{2}=Z^{2}\left(1-0.02 Z^{1 / 3}\right) .
$$

In particular, for the tin target $(Z=50): \sigma_{0}=1.88$ barn; $\Delta \sigma=-0.14$ barn; $\quad \sigma=1.74$ barn. For the uranium target $(Z=92): \sigma_{0}=6.38$ barn; $\Delta \sigma=-0.58$ barn; $\sigma=5.8$ barn.

Here $\sigma_{0}$ is the cross-section calculated for the point-like Coulomb centre, $\Delta \sigma$ is the correction to the cross-section due to the finite radius of the target nucleus.

2. Now let us consider the process of Coulomb dissociation of the hypernucleus ${ }^{6} \mathrm{He}_{\Lambda}$ into ${ }^{5} H e_{\Lambda}$ and the neutron: ${ }^{6} H e_{\Lambda}+Z \rightarrow{ }^{5} H e_{\Lambda}+n+Z\left(z=2, M=M\left({ }^{6} H e_{\Lambda}\right)=5.78 \mathrm{GeV} / c^{2}\right.$, $\left.m_{1}=m\left({ }^{5} H e_{\Lambda}\right)=4.84 \mathrm{GeV} / c^{2}, m_{2}=m_{n}=939 \mathrm{MeV} / c^{2}\right)$.

The data on the binding energies of the $\Lambda$-hyperon in the hypernuclei ${ }^{6} \mathrm{He}_{\Lambda}$ and ${ }^{5} \mathrm{He} e_{\Lambda}$ [6] and on the masses of their nucleon bases (ordinary nuclei ${ }^{5} \mathrm{He}$ and ${ }^{4} \mathrm{He}$ [7]) lead to the following estimation of the binding energy of the neutron in the hypernucleus ${ }^{6} H e_{\Lambda}: \varepsilon_{\text {bin }}^{(n)}=(0.23 \pm 0.13)$ $\mathrm{MeV}$. Taking $\gamma=6$ and $\varepsilon_{\text {bin }}^{(n)} \approx 0.15 \mathrm{MeV}$, we have:

$$
\chi \approx 1.43 Z^{1 / 3} ; \quad \Delta B \approx 0.5 \chi: \quad Z_{\mathrm{eff}}^{2} \approx Z^{2}\left(1-0.05 Z^{1 / 3}\right) .
$$

In the case of the tin target $(Z=50): \quad \sigma_{0}=0.74$ barn; $\Delta \sigma=-0.14$ barn; $\sigma=0.6$ barn. For the uranium target $(Z=92)$ : $\sigma_{0}=2.50$ barn; $\Delta \sigma=-0.54$ barn; $\sigma=1.96$ barn.

So, for the considered cases (especially - for ${ }^{6} H e_{\Lambda}$ ) the correction $\Delta \sigma$ to the Coulomb dissociation cross-section, emerging due to the finite size of the target, proves to be rather substantial.

\section{References}

[1]V.L. Lyuboshitz, Yad. Fiz. 51, 1013 (1990) [ Sov. Jour. Nucl. Phys. 51, 648 (1990)]

[2]V.B. Berestetsky, E.M. Lifshitz and L.P. Pitaevsky, Quantum Electrodynamics (in Russian) (Nauka, Moscow, 1980), §82 [transl. Pergamon Press, Oxford, 1982, 2nd ed.]

[3]V.L. Lyuboshitz and V.V. Lyuboshitz, Yad. Fiz. 70, 1663 (2007) [ Phys. At. Nucl. 70, 1317 (2007) ]

[4]V.L. Lyuboshitz and V.V. Lyuboshitz, Nucl. Phys. A 790, p. 294c (2007) - in Proceedings of the 18-th International Few-Body Conference ( FB18, Santos, Brazil, August 21 - 26, 2006 ) .

[5]G. Bohm et al., Nucl. Phys. B 4, 412 (1968)

[6]M. Juric' et al., Nucl. Phys. B 52, 1 (1973)

[7]H.A. Bethe and Ph. Morrison, Elementary nuclear theory (John Wiley \& Sons, Inc., New York London, 1956), Appendix . 